\title{
Neonatal exposure to androgens dynamically alters gut microbiota architecture
}

\author{
Alexia Barroso 1,2,*, Jose Antonio Santos-Marcos²,3*, Cecilia Perdices-Lopez 1,2, Ana Vega-Rojas 2,3, \\ Miguel Angel Sanchez-Garrido1,2, Yelizabeta Krylova2,3, Helena Molina-Abril4, Claes Ohlsson', \\ Pablo Perez-Martinez2,3, Matti Poutanen5,6, Jose Lopez-Miranda2,3, Manuel Tena-Sempere 1,2,6 and \\ Antonio Camargo 1 2,3
}

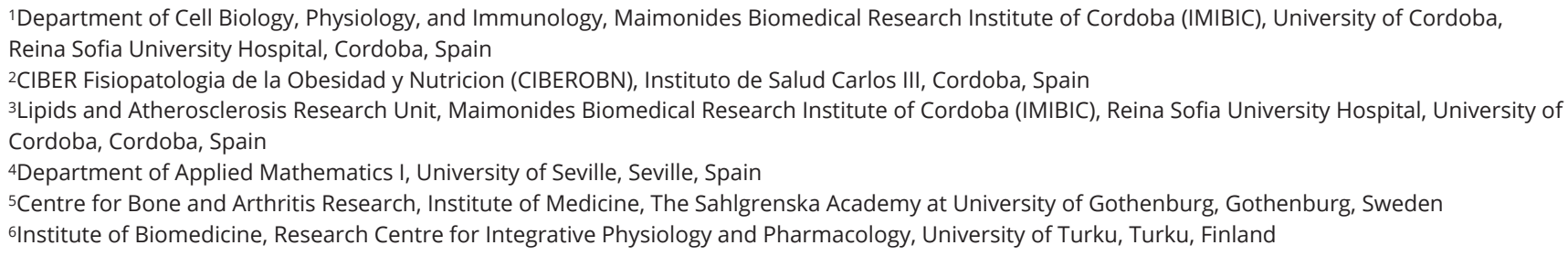

\begin{abstract}
Gonadal steroids strongly contribute to the metabolic programming that shapes the susceptibility to the manifestation of diseases later in life, and the effect is often sexually dimorphic. Microbiome signatures, together with metabolic traits and sex steroid levels, were analyzed at adulthood in neonatally androgenized female rats, and compared with those of control male and female rats. Exposure of female rats to high doses of androgens on early postnatal life resulted in persistent alterations of the sex steroid profile later on life, namely lower progesterone and higher estradiol and estrone levels, with no effect on endogenous androgens. Neonatally androgenized females were heavier ( $10 \%$ at early adulthood and $26 \%$ at adulthood) than controls and had impaired glucose homeostasis observed by higher AUC of glucose in GTT and ITT when subjected to obesogenic manipulations. Androgenized female displayed overt alterations in gut microbiota, indicated especially by higher Bacteroidetes and lower Firmicutes abundance at early adulthood, which disappeared when animals were concurrently overfed at adulthood. Notably, these changes in gut microbiota were related with the intestinal expression of several miRNAs, such as miR-27a-3p, miR-29a-5p, and miR-100-3p. Our results suggest that nutritional and hormonal disruption at early developmental periods not only alters the metabolic programming of the individual later in life but also perturbs the architecture of gut microbiota, which may interact with the host by a cross-talk mediated by intestinal miRNAs; phenomena that may contribute to amplify the metabolic derangement caused by obesity, as seen in neonatally androgenized female rats.
\end{abstract}

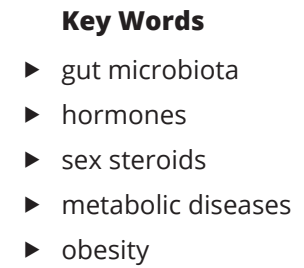

Journal of Endocrinology (2020) 247, 69-85 (c) 2020 Society for Endocrinology Published by Bioscientifica Ltd. Printed in Great Britain 


\section{Introduction}

Sex steroids are key metabolic regulators in different tissues, with proven roles in the control of food intake and energy homeostasis, whose deregulation is frequently linked to the manifestation of diseases (Mauvais-Jarvis et al. 2013). In addition, the incidence of metabolic diseases and their co-morbidities is sexually dimorphic, and varies depending on the gonadal status; for example, increases after menopause (Mauvais-Jarvis 2015).

However, the influence of gonadal factors is rather complex. For instance, male hypogonadism is considered a risk factor for the development of cardiovascular complications associated to type 2 diabetes, suggesting that low testosterone in adult men is detrimental for metabolic and cardiovascular homeostasis (Rao et al. 2013). By contrast, androgen excess in women, a characteristic of polycystic ovary syndrome (PCOS), is associated to insulin resistance, which also contributes to the excessive ovarian androgen production (Witchel et al. 2019).

Early metabolic programming by sex steroids also contributes to define differences in susceptibility to later development of the metabolic disease. Inappropriate exposures to sex steroids during early maturational periods (e.g. excessive androgenization in utero) have been linked to the development of insulin resistance and PCOS (Witchel \& Tena-Sempere 2013). Hence, gonadal hormones are likely to influence metabolic homeostasis via multiple, frequently redundant, regulatory systems, and can be considered as genuine modifiers of metabolic homeostasis, which may contribute to generate differences in susceptibility for the developing of cardiometabolic disease (Faulkner \& Belin de Chantemele 2019).

Gut microbiota is now recognized as an organ integrated in the metabolism of the host (Tremaroli \& Backhed 2012), and it has been proposed that alteration of the gut microbiota structure may trigger the development of cardiometabolic diseases (Jie et al. 2017). Notably, sex hormones appear to be one of the factors driving gut microbiota differences between males and females, which may also contribute to the sexual dimorphism in disease susceptibility (Cross et al. 2018). In addition, it has been shown that the gut microbiota structure is influenced by the combined effects of sex, gonadal hormones, and obesity, with specific abnormalities in women with PCOS (Insenser et al. 2018).

In this study, we aimed to explore, using suitable preclinical (rat) models, the putative role of sex steroid milieu at early developmental periods, alone or in combination with obesogenic insults later in life, as persistent modifiers of gut microbiota architecture, in the context of development of metabolic alterations. In order to shed light on the putative mechanisms for the integration between gut microbiota and host, we also explored the cross-talk between gut microbiota and the host through regulation of the expression of miRNAs in small and large intestine, as intestinal miRNAs are increasingly recognized as potential decoders of the impact of dysbiosis into cardiometabolic diseases (Liu et al. 2016, Serino 2016).

\section{Materials and methods}

\section{Animals and diets}

Wistar male and female rats bred in the vivarium of the University of Cordoba were used. The animals were maintained at $22 \pm 1{ }^{\circ} \mathrm{C}$ under constant conditions of light $(14 \mathrm{~h})$ with free access to water. The experimental animals were fed a control diet (CD), D12450B (10, 20, and $70 \%$ calories from fat, protein, and carbohydrate, respectively), or a high fat diet (HFD), D12451 $(45 \%, 20 \%$, and $35 \%$ calories from fat, protein and carbohydrate, respectively; Research Diets Inc., New Brunswick, NJ, USA).

\section{Experimental design}

On postnatal day (PND)-1, males and female pups were cross-fostered and reared in two different litter sizes: small litters (SLs: 4 pups per litter; as a model of postnatal overnutrition) or normal litters (NLs: 12 pups per litter), as extensively described previously (Castellano et al. 2011, Sanchez-Garrido et al. 2013, 2014). Immediately afterwards, subsets of female pups of the two litter sizes were androgenized by a single s.c. injection of a bolus of $1.25 \mathrm{mg}$ of testosterone propionate dissolved in olive oil $(100 \mu \mathrm{L})$, whereas the remaining female pups were injected with vehicle (olive oil), following previously validated protocols (Pinilla et al. 2002). After weaning on PND-23, groups of androgenized or vehicle-treated females, as well as males, were randomly pooled within each category (androgenization or vehicle; NL or SL) to avoid the bias of differences in body weight (BW) between the different subgroups, and were housed in a number of four-five rats per cage. From weaning onwards, the groups of SL rats (either males, vehicle-treated or androgenized females) were fed with an HFD, while the NL groups received a CD; all animals had access to food ad libitum. 
Analyses were applied at two age points, on PND-50 and PND-150, representative of young adult (PND-50) or adult rats (PND-150), in order to check the short- and long-term the impact of the different stressors (neonatal androgenization, and obesogenic dietary patterns). Rats of both age-groups were killed by decapitation, and trunk blood and fecal and tissue samples were collected for analyses. These analyses included phenotypic indices and biochemical/hormonal parameters in serum, as well as fecal and intestinal samples. In intact females, sampling was carried out at the same stage of the ovarian cycle, namely diestrus- 1 , and was conducted between 9:00 $\mathrm{h}$ and noon to avoid the potential interference of circadian variations (Castellano et al. 2011). Small and large intestine sections were dissected and fecal samples were obtained from the different study groups directly from stool expulsion stimulated by manual handling. The samples obtained were frozen in liquid nitrogen and stored at $-80^{\circ} \mathrm{C}$ until analysis. All the experimental protocols were approved by Cordoba University Ethical Committee of animal experimentation and conducted in accordance with the European Union guidelines for the use of experimental animals.

\section{Phenotypic indices and hormonal measurements}

Body weight (BW) and basal blood glucose levels were recorded in all the experimental groups, at the two agepoints (PND-50 and -150); glucose levels were determined after overnight fasting. In addition, serum level of leptin was assayed at the same ages by a double-antibody RIA, using the kit provided by EMD MILLIPORE. The limit of sensitivity of the assay was $0.801 \mathrm{ng} / \mathrm{mL}$, and the intra- and inter-assay coefficients of variation were less than 4 and 9\%, respectively. At the two ages, the serum levels of ghrelin were also measured using a Bio-Plex Rat Diabetes Assay, provided by Bio-Rad Laboratories. The limit of sensitivity of the assay was $0.3 \mathrm{pg} / \mathrm{mL}$, and the intra- and inter-assay CVs were 4 and $4 \%$, respectively. In all experimental groups, sex steroid plasma levels were determined at PND-50 and -150, using a sensitive gas chromatography-tandem mass spectrometry method, thoroughly validated as described in previous references (Nilsson et al. 2015, Velasco et al. 2019).

\section{Glucose tolerance tests and insulin tolerance tests}

Rats of all experimental groups were subjected to glucose tolerance tests (GTTs) at the two age groups indicated previously. Rats were fasted overnight and subsequently received an intraperitoneal (ip) bolus of glucose $(1 \mathrm{~g} / \mathrm{kg}$ BW). Glucose levels were determined in blood before (0) and at 20,60, and 120 min post-administration. After complete recovery 1 week later, insulin sensitivity was assessed by an insulin tolerance tests (ITT). Rats were fasted overnight, following an ip injection of 1UI insulin (Sigma-Aldrich) per kg body weight. Blood glucose levels were measured before (0) and at 20, 60, and 120 min after insulin administration. Integral glucose changes levels were estimated as area under the curve (AUC), during the 120 min period after glucose or insulin administration, as calculated by the trapezoidal method. All glucose concentrations were measured using a handheld glucometer (ACCU-CHECK Aviva; Roche Diagnostics).

\section{Intestinal microbiota analysis}

DNA extraction from feces was performed using the QIAamp DNAStool Mini Kit Handbook (QIAGEN), following the manufacturer's instructions.

The microbiota composition analysis of the fecal samples was performed on a MiSeq Illumina platform (Illumina, San Diego, CA, USA), according to the manufacturer's instructions. Briefly, PCR (PCR) was performed using $0.2 \mu \mathrm{M}$ of each one of the primer 5'-TCGTCGGCAGCGTCAGATGTGTATAAGAGACAG-3' and 5'-GTCTCGTGGGCTCGGAGATGTGTATAA GAGACAG-3' (Klindworth et al. 2013) to generate amplicons containing the hypervariable region V3 of the 16s rRNA gene, KAPA HiFi HotStart ReadyMix (KAPABIOSYSTEMS), and $1.25 \mu \mathrm{L}$ of extracted DNA (5 $\mathrm{ng} / \mu \mathrm{L}$ in $10 \mathrm{mM}$ Tris $\mathrm{pH}$ 8.5), using the following cycle parameters: $3 \mathrm{~min}$ denaturation at $95^{\circ} \mathrm{C}$ followed by 25 cycles $\left(30 \mathrm{~s}\right.$ at $95^{\circ} \mathrm{C}, 30 \mathrm{~s}$ at $60^{\circ} \mathrm{C}$, $30 \mathrm{~s}$ at $72^{\circ} \mathrm{C}$ ) and a final extension at $72^{\circ} \mathrm{C}$ for $5 \mathrm{~min}$. The amplicon purification was performed using Agentcourt AMPure XP beads (Beckman Coulter).

A second PCR reaction attaches dual indices and Illumina sequencing adapters using the Nextera XT Index Kit. This PCR was performed with a KAPA HiFi HotStart ReadyMix (KAPABIOSYSTEMS), $5 \mu \mathrm{L}$ of the previous amplicon, $5 \mu \mathrm{L}$ of each Nextera XT Index Primer 1 (N7xx) and $5 \mu \mathrm{L}$ of each Nextera XT Index Primer 2 (S5xx), using the following cycle parameters: 3 min denaturation at $95^{\circ} \mathrm{C}$ followed by eight cycles $\left(30 \mathrm{~s}\right.$ at $95^{\circ} \mathrm{C}, 30 \mathrm{~s}$ at $55^{\circ} \mathrm{C}, 30 \mathrm{~s}$ at $72^{\circ} \mathrm{C}$ ), and a final extension at $72^{\circ} \mathrm{C}$ for $5 \mathrm{~min}$. The PCR product purification was performed using Agentcourt AMPure XP beads (Beckman Coulter). Raw data are available at NCBI Sequence Read Archive (SRA) under the accession number PRJNA631334. Sequencing 
data were analyzed and visualized using QIIME 2 v. 2019.7 (Bolyen et al. 2019). Demultiplexed single-end reads containing V3 hypervariable region were truncated at 212 bp (Quality score median $>30$ ), and denoised using DADA2 method (Callahan et al. 2016).

After filtering, the high-quality reads of the 64 samples ( $n=8$ for each group) ranging from 170,919 to 18,682 sequence counts, and rarefaction depth was established at 18,500 sequence counts. Bacterial richness and diversity across the samples were calculated using the observed OTUs and Shannon indexes (Hammer et al. 2001). Principal component analysis of community structure (beta-diversity) was done using the unweighted and weighted UniFrac distance metrics (Lozupone \& Knight 2005) and analyzed by permutational multivariate ANOVA (PERMANOVA). Taxonomy was assigned to the high-quality reads using q2-feature-classifier (Bokulich et al. 2018) with a sequence identity threshold of $99 \%$ interrogating the sequences with the Greengenes database (13_8) (McDonald et al. 2012). To be consistent with the $16 \mathrm{~S}$ rRNA obtained taxonomic data, only taxa in the bacteria domain were included in the statistical analysis.

The relative taxonomic abundance was measured as the proportion of reads over the total in each sample assigned to a given taxonomy. To exclude bacterial taxa that were not present in the majority of samples, a cutoff for exclusion was fixed; only bacterial taxa containing sequence reads in at least $75 \%$ of total samples were considered. Linear discriminant analysis (LDA) effect size (LEfSe) (http://huttenhower.sph.harvard.edu/galaxy/) was used to compare groups at baseline and visualize the results using taxonomic bar charts and cladograms (Segata et al. 2011).

\section{RNA isolation from small and large intestine}

Frozen tissue was ground to a fine powder in liquid nitrogen, using a mortar and pestle. RNA was isolated with the commercial kit Direct-zol ${ }^{\mathrm{TM}}$ RNA MiniPrep Plus (Zymo Research Corp., CA, USA), and quantified using the spectrophotometer v3.5.2 Nanodrop ND-1000 (Nanodrop Technologies, Cambridge, UK).

\section{miRNA expression analysis}

miRNA expression profiles were generated using the SurePrint Rat miRNA Microarrays, Rat miRNA 8x15K Microarray (Release 21.0) (Agilent Technologies Inc.).
RNA samples of each experimental group were pooled and labeled using the miRNA Labeling and Hyb Kit (Agilent Technologies Inc.), according to the manufacturer's instructions. Hybridization was performed using this latter kit, also according to the manufacturer's instructions. Microarray images of each slide were obtained with a Gene Pix 4000B scanner (Axon Instruments, Union City, CA, USA). Image quantization was performed using Agilent Feature Extraction Software (Agilent Technologies Inc.). Raw microarray data were analyzed using the limma R package (Smyth 2005). Spots with foreground mean and median differing by more than 50 were filtered out and data quality was checked using limma tools. Background correction was performed using saddle-point approximation in the normal-exponential convolution method Normexp (Ritchie et al. 2007). Then within arrays Print-tip loess (Yang et al. 2001) and between arrays quantile were used for normalization. Finally, replicate spots in the array data were averaged.

\section{Software for miRNA analysis}

To identify the role of selected miRNAs in the cellular processes, we performed an analysis using the DIANA tools V.3. DIANA-miRPath is a web-server (http://diana. imis.athena-innovation.gr/DianaTools/index.php), which provides accurate statistics and can accommodate advanced pipelines. miRPath can utilize predicted miRNA targets (in CDS or 3'-UTR regions) provided by the DIANAmicroT-CDS algorithm or even experimentally validated miRNA interactions derived from DIANA-TarBase (Vlachos et al. 2015).

\section{Statistical analysis}

PASW statistical software package, version 20.0 (IBM Inc.), was used for statistical analyses of the data. We used One-way ANOVA to test the differences between animal's groups. We used ANOVA for repeated measures for the time-course glucose levels analysis in the GTT and ITT with time as intra-individual factor and animal's group as inter-individual factor. Post hoc was performed by Bonferroni multiple comparisons test. Pearson's correlation test was used to evaluate the relationship between miRNAs intestinal expression and bacterial taxa abundance. Data are presented as mean \pm s.E.M. $P$-values $<0.05$ were considered statistically significant in all the statistical analyses. https://joe.bioscientifica.com

https://doi.org/10.1530/JOE-20-0277 (c) 2020 Society for Endocrinology Published by Bioscientifica Ltd. Printed in Great Britain 


\section{Results}

\section{Effect of androgenization on sex steroid profile according to the nutritional status}

In order to evaluate the early metabolic programming by sex steroids on the susceptibility to later development of metabolic disease, female rats were subjected to neonatal androgenization by single injection of a bolus of testosterone propionate (1.25 mg, s.c.) on PND-1. By measuring testosterone, dihydrotestosterone and androstenedione later on life, we did not observe differences in androgen levels between androgenized and not androgenized females, with the exception of $49.7 \%$ lower androstenedione levels in androgenized females $(P=0.014)$ under NL-CD conditions at PND-150. As expected, in none of the female study groups the androgen levels reached that of the males, with the exception of testosterone and androstenedione for which the difference between the males and androgenized females under NL-CD condition at PND-50 did not reach the statistical significance. Of importance, neonatal androgenization reduced progesterone levels in the females at PND-50 and -150 in both NL-CD (0.22 and 0.15-fold change) and SL-HFD (0.19 and 0.28-fold change) conditions, compared to control females (all, $P$-values < 0.05 ). Thus, the progesterone levels in androgenized females turned toward that measured in males. Interestingly, estradiol (E2) levels were markedly (16.61 and 8.91-fold change, $P<0.001$ and $P=0.018$, respectively) increased in females at PND-50 as a consequence of neonatal androgenization, in both NL-CD and SL-HFD conditions, whereas no statistically significant differences were found between the groups at PND-150. Moreover, estrone (E1) level was markedly higher in androgenized females at PND-50, in both NL-CD (26.28-fold change, $P=0.009$ ) and SL-HFD (high levels vs not detected), and at PND-150 in SL-HFD (31.89-fold change, $P=0.011$ ) conditions compared to intact females. As expected, only very low or non-detectable level of E2 and E1 was measured in the males (Fig. 1).

\section{Early manipulation of the sex steroid milieu impairs energy balance and glucose homeostasis}

Neonatal androgenization increased BW of the females measured at 50 and 150 days, in both NL-CD and SL-HFD conditions compared to intact females. We found a grossly similar profile in leptin levels in both intact and androgenized females, but increased leptin levels were observed in androgenized females compared to intact rats under SL-HFD at PND-150. In these rats, leptin levels were close to the ones observed in males. By contrast, ghrelin levels were lower in androgenized females than males (NL-CD and SL-HFD) and intact females (NL-CD) at PND-50, with no differences between groups at PND-150 (Supplementary Fig. 1, see section on supplementary materials given at the end of this article).

In addition, GTT and ITT were applied to the experimental groups to assess the potential alterations in glucose homeostasis. We found that androgenized female rats at PND-50 displayed an AUC of glucose higher than intact females, which reached the statistical
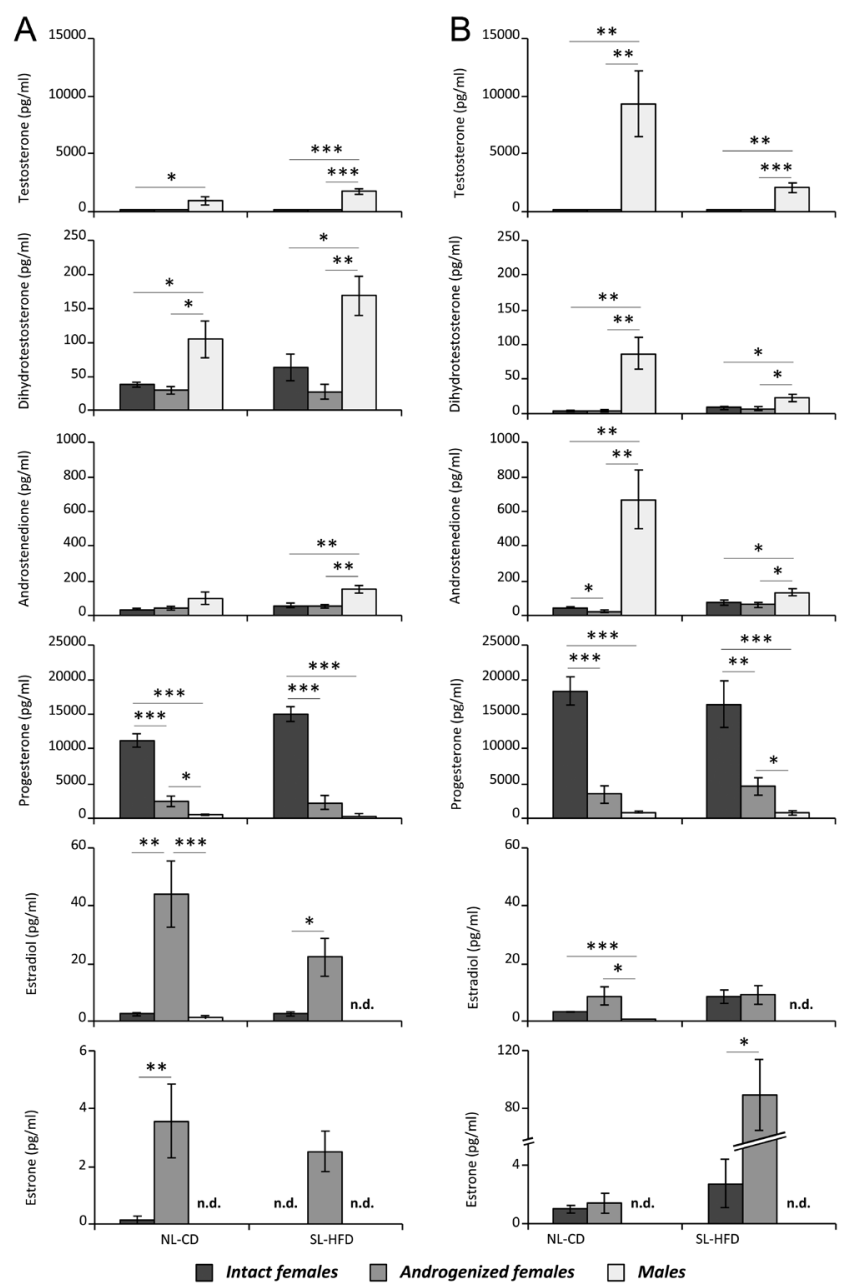

Figure 1

Serum sex steroid levels in intact (non-androgenized) animals and androgenized females, under normal feeding (NL-CD) and postnatal overfeeding (SL-HFD) at PND-50 (A) and PND-150 (B). NL-CD, normal litter, control diet; SL-HFD, small litter, high fat diet; PND, postnatal day. $\star P$-value $<0.05, * * P$-value $<0.01$, and $* * * P$-value $<0.001$ in the one-way ANOVA statistical analysis. n.d., not detectable. 
A
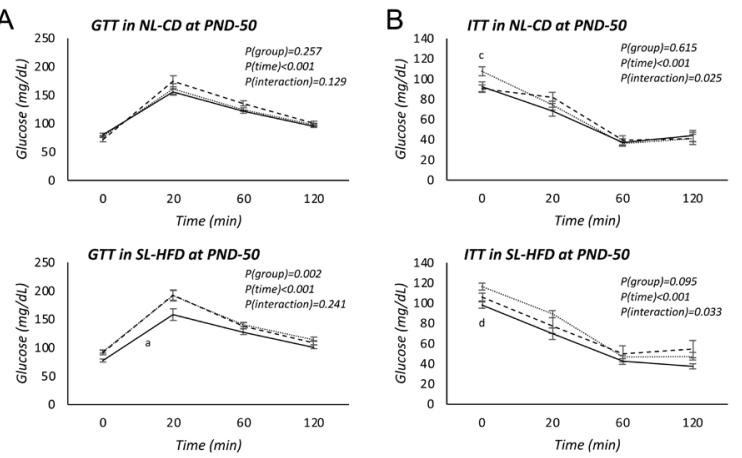

GTT in NL-CD at PND-150

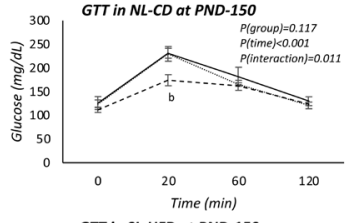

TT in SL-HFD at PND-150

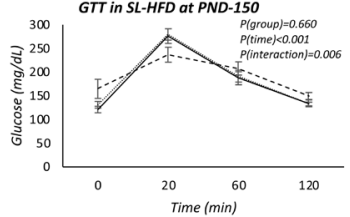

- Intact females
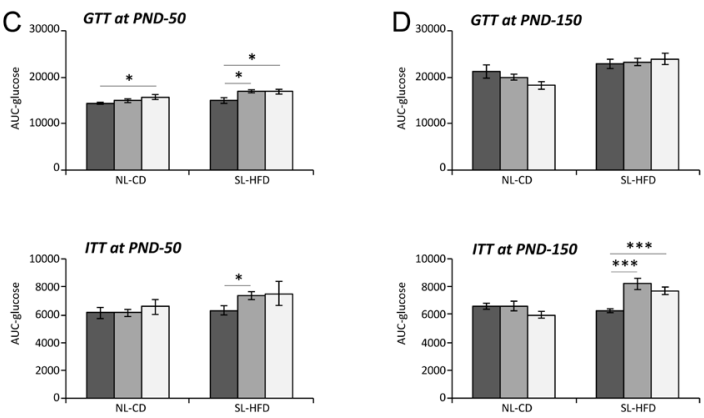

$\square$ Intact females $\quad \square$ Androgenized females $\quad \square$ Males

\section{Figure 2}

Metabolic parameters in intact animals and androgenized females. Time-course profiles and area under curve of serum glucose in glucose and insulin tolerance tests, under normal feeding (NL-CD) and postnatal overfeeding (SL-HFD). NL-CD, normal litter, control diet; SL-HFD, small litter, high fat diet; PND, postnatal day. Glucose tolerance test (GTT) was performed in young adult (<PND-50) and adult (between PND-120 and PND-150) animals. After complete recovery 1 week later, insulin tolerance tests (ITTs) was assessed. Time-course profiles (A and B) ANOVA for repeated measures statistical analysis. (a) Intact females lower than androgenized females $(P=0.004)$ and males $(P=0.010)$. (b) Males lower than intact females $(P=0.014)$ and androgenized females $(P=0.017)$ at time point of $20 \mathrm{~min}$. (c) Androgenized females higher than males $(P=0.031)$ and a trend with intact females $(P=0.064)$ at time point of 0 min. (d) Androgenized females higher than intact females $(P=0.004)$ at time point of 0 min. (e) Androgenized females higher than males $(P=0.012)$ at time point of $20 \mathrm{~min}$. (f) Intact females lower than androgenized females $(P=0.001)$ and males $(P=0.009)$. $(\mathrm{g})$ Intact females lower than androgenized females $(P<0.001)$ and males $(P=0.022)$ at time point of $60 \mathrm{~min}$. (h) Intact females lower than androgenized females $(P=0.005)$ and a trend with males $(P=0.076)$ at time point of $120 \mathrm{~min}$. Area under curve (AUC) ( $C$ and D) One-way ANOVA statistical analysis. $\star P$-value $<0.05$ and $* * * P$-value $<0.001$

(c) 2020 Society for Endocrinology Published by Bioscientifica Ltd. Printed in Great Britain significance under SL-HFD, but not under NL-CD (Fig. 2). In fact, the analysis of the time-course glucose levels on the GTT showed higher glucose levels (indicative of lower glucose tolerance) in androgenized females (and males) at PND-50 than in intact females (Fig. 2). In addition, we observed in the ITT a higher AUC of glucose (indicative of lower insulin sensitivity) in androgenized females than in intact females under SL-HFD, but not under NL-CD, at both PND-50 and PND-150 (Fig. 2). Moreover, time-course glucose levels at 0 min on the ITT showed higher glucose levels in androgenized females than intact females at PND-50 under both NL-CD and SL-HFD conditions. In addition, glucose levels on the ITT showed higher glucose levels of androgenized females (and males) than intact females at PND-150 under SL-HFD condition, whereas no differences were shown under NL-CD (Fig. 2).

\section{Impact of early androgenization on gut microbiota diversity}

We evaluated whether the disruption on the early metabolic programming by neonatal androgenization impacts on the gut microbiota architecture. These studies showed that there were no significant differences in the $\alpha$-diversity measured by the Shannon and observed OTUs indexes, and by the phylogenetic diversity (Faith) between androgenized and intact females at any age (PND-50 nor PND-150) and nutritional condition (NL-CD and SL-HFD). However, there was a higher $\alpha$-diversity at PND-50 of the bacterial community in androgenized females than in males under NL-CD condition, but these differences were not found when animals were raised under SL-HFD condition. By contrast, lack of differences or only trends were found between androgenized females and males, at PND-150 under both NL-CD and SL-HFD conditions, with the exception of Observed OTUs index (Supplementary Table 1).

In term of bacterial $\beta$-diversity, we found significant unweighted UniFrac distances among androgenized, intact female and male groups (all $P$ and $Q$ values < 0.05), but no differences at PND-150 in the SL-HFD groups between androgenized and intact females. In contrast, we found significant differences in weighted UniFrac distances among the main groups (androgenized, intact females and males) under NL-CD, and between androgenized females and intact females under SL-HFD condition. However, no differences were found between androgenized females and males under SL-HFD condition (Supplementary Table 1). 


\section{Journal of Endocrinology \\ A Barroso, J A Santos- \\ Marcos et al. \\ Impact of early androgenization on gut microbiota composition at PND-50}

Hormonal disruption and gut microbiota
Further, we analyzed the differences in the microbiota structure by LEfSe analysis (Fig. 3 and Supplementary Figs 2,3). The data showed that in NL-CD animals at PND-50 the gut microbiota from androgenized females was characterized by higher abundance of Bacteroidetes phylum and by a reduced abundance of Firmicutes and Euryarchaeota phyla compared to intact females. At genus level, we observed that androgenization increased the abundance of Bacteroides, Helicobacter and CF231 genera in addition to one unknown genus from $524-7$ family. The androgenization, furthermore, reduced the abundance of Methanobrevibacter, Desulfovibrio and p-75-a5 genera, in addition to an unknown genus from
Desulfovibrionaceae family. In contrast, when animals were raised under SL-HFD conditions, no differences in the majority phyla, Firmicutes and Bacteroidetes, were found between androgenized and intact females, but we found that androgenization reduced the Euryarchaeota, Verrucomicrobia, and Cyanobacteria phyla. We observed a lower Firmicutes/Bacteroidetes (F/B) ratio in androgenized females than intact females $(P=0.022)$ at PND-50 under NL-CD condition, whereas no differences were observed under SL-HFD condition (Supplementary Table 2).

Atgenuslevel, we found that androgenizationincreased Clostridium (Clostridiaceae), Clostridium (Lachnospiraceae), and Lactobacillus. We also observed that androgenization reduced Ruminococcus (Ruminococcaceae), Oscillospira, Clostridium (Peptostreptococcaceae), Methanobrevibacter, Phascolarctobacterium, Flexispira, Dehalobacterium, Alistipes,
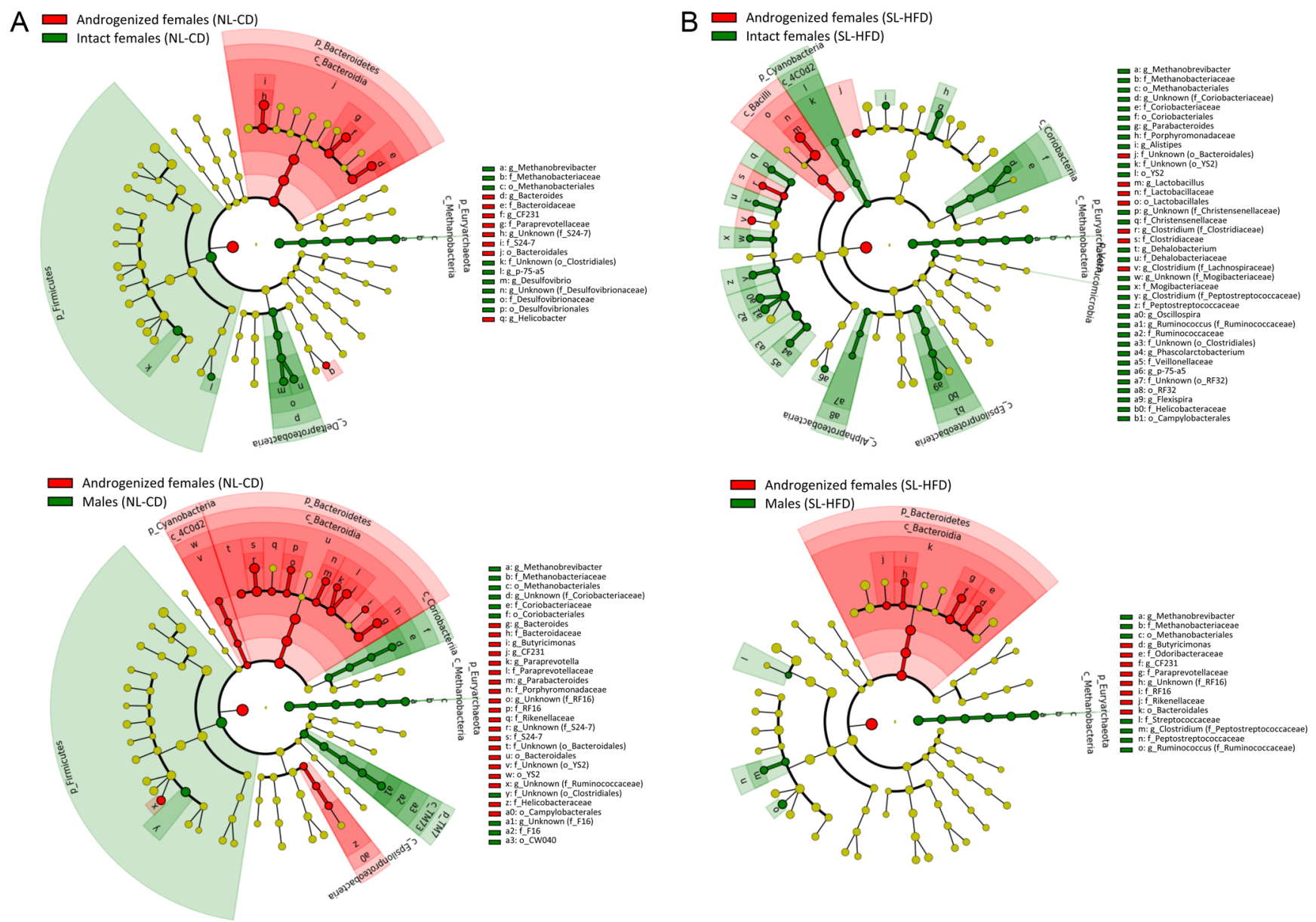

Figure 3

LEfSe analysis between androgenized females and intact females (and males) under normal feeding (A) and postnatal overfeeding (B) conditions at PND-50. Cladogram representing the taxonomic hierarchical structure of the identified differences between groups using Linear discriminant analysis effect size (LEfSe). Each filled circle represents one phylotype. Red, bacterial taxa statistically overrepresented in androgenized females; green, bacterial taxa overrepresented in females (or males). Phylum and class are indicated in their names on the cladogram and the order, family, or genus are given in the key. A full colour version of this figure is available at https://doi.org/10.1530/JOE-20-0277. 
p-75-a5, and Parabacteroides genera, in addition to 3 unknown genera from unknown from Christensenellaceae, Coriobacteriaceae, and Mogibacteriaceae families.

\section{Impact of early androgenization on gut microbiota composition at PND-150}

Analyses of the differences in community structure by LEfSe analysis at PND-150 (Fig. 4 and Supplementary Figs $4,5)$ revealed that, in NL-CD animals, androgenization increased Cyanobacteria phylum, and reduced Euryarchaeota and Actinobacteria phyla, but no differences in the majority phyla, Firmicutes and Bacteroidetes, were found as compared with intact females. At genus level, we found that androgenization increased CF231 genus in addition to three unknown genera from the WCHB1-25, Elusimicrobiaceae, and RF16 families, whereas it reduced
Methanobrevibacter and Turicibacter, in addition to two unknown genera from S24-7, and Coriobacteriaceae families. In turn, in SL-HFD animals, we found that androgenization increased Bacteroidetes phylum, in addition to discrete changes at genus level, as an increase in Alistipes and Odoribacter genera, and differences in two unknown additional genera from RF16 (increase), and Coriobacteriaceae (decrease) families. Moreover, we observed no differences in $\mathrm{F} / \mathrm{B}$ ratio between androgenized and intact females at PND-150 under both NL-CD and SL-HFD conditions (Supplementary Table 2).

\section{Microbiota putatively modulates host metabolism via changes in miRNAs}

In order to evaluate the cross-talk between gut microbiota and the changes in sex hormones and obesity of the host,

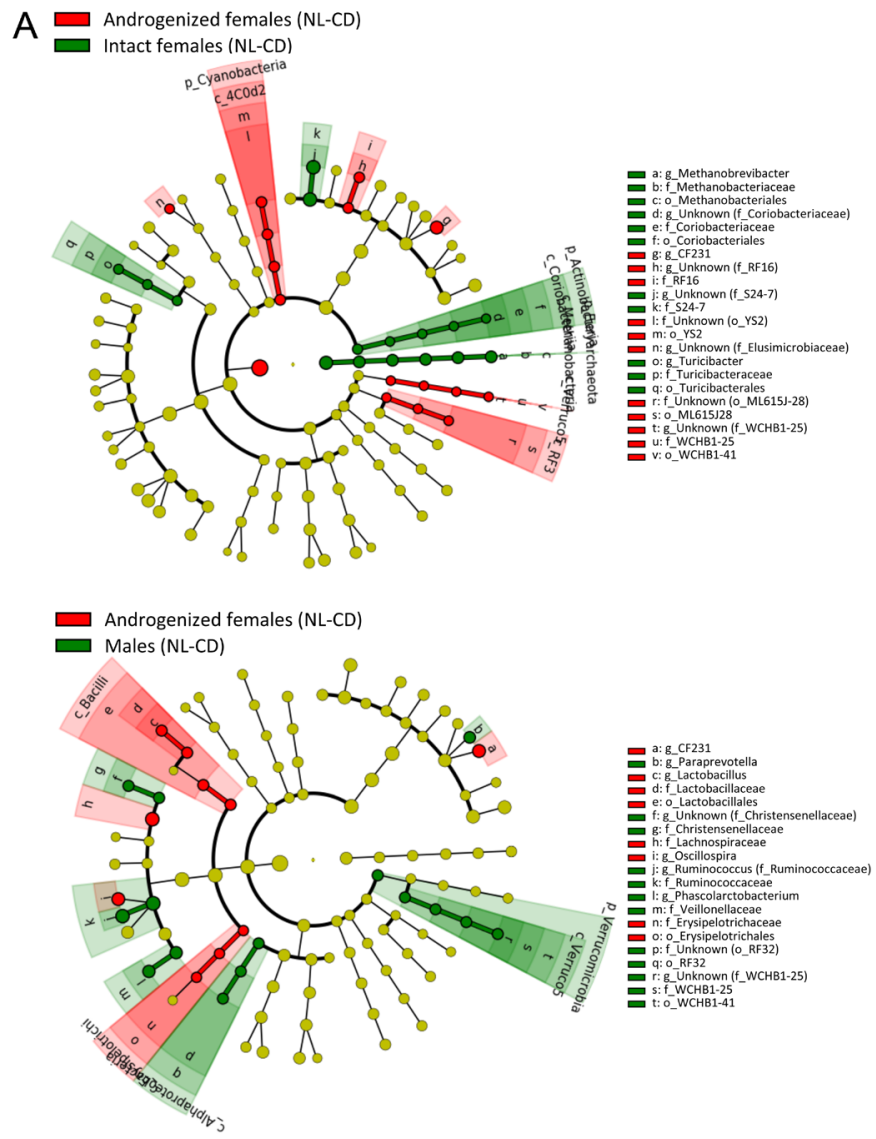

$\mathrm{B} \square$ Androgenized females (SL-HFD)
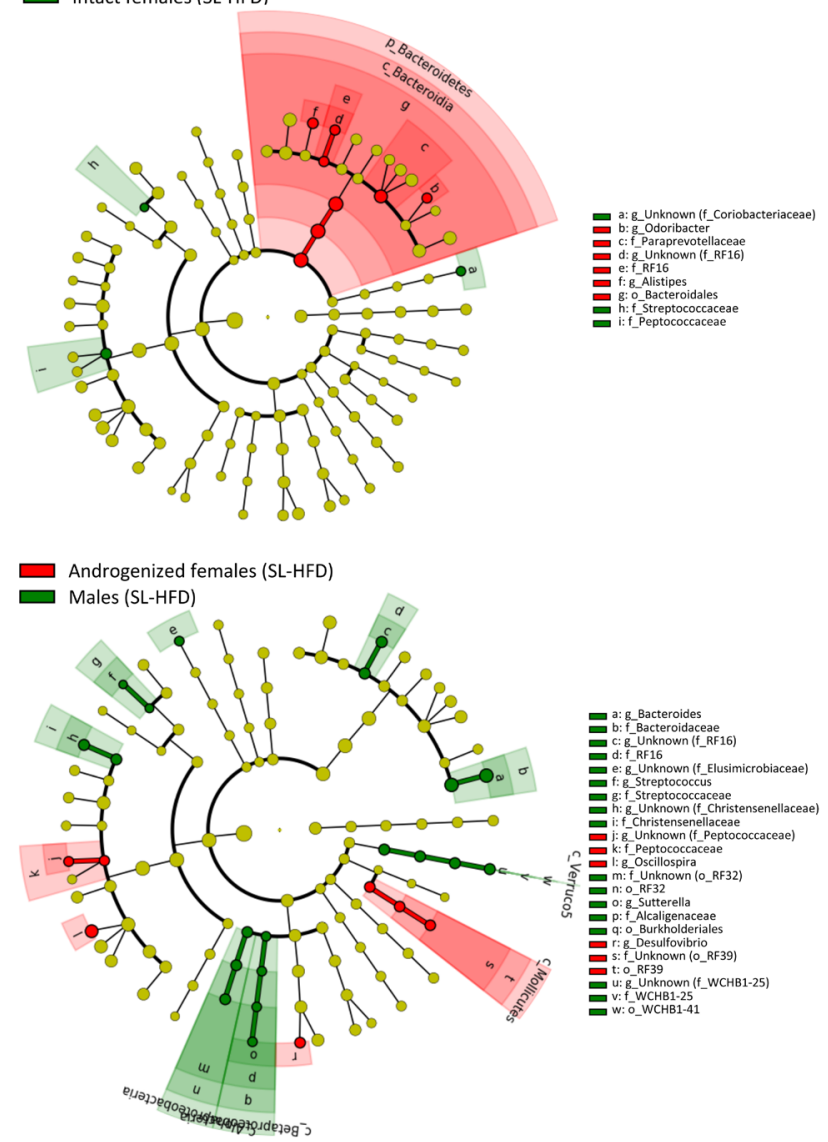

Figure 4

LEfSe analysis between androgenized females and intact females (and males) under normal feeding (A) and postnatal overfeeding (B) conditions at PND-150. Cladogram representing the taxonomic hierarchical structure of the identified differences between groups using Linear discriminant analysis effect size (LEfSe). Each filled circle represents one phylotype. Red, bacterial taxa statistically overrepresented in androgenized females; green, bacterial taxa overrepresented in females (or males). Phylum and class are indicated in their names on the cladogram and the order, family, or genus are given in the key. A full colour version of this figure is available at https://doi.org/10.1530/JOE-20-0277. 
we analyzed the relationship between the bacterial taxa identified by LEfSe analysis according to sex hormones and obesity, and the expression levels of the miRNAs in small and large intestine. First of all, microarray analysis testing 758 miRNAs detected the expression of 60 miRNAs in large intestine and 103 miRNAs in small intestine of the animals in our experimental conditions. Of note, the expression of 25 of these miRNAs was detected in both large and small intestine. We performed a correlation analysis between the bacterial taxa identified by LEfSe analysis and the intestinal expression of miRNAs. From this correlation analysis, we selected 30 miRNAs in small intestine and 23 miRNAs in large intestine (4 miRNAs expressed in both large and small intestine showed a relationship with any bacterial taxa identified by LEfSe analysis) in which Pearson correlation coefficient was $>0.9$ or $<-0.9$, and a $P$-value $<0.01$ (Figs 5,6 and
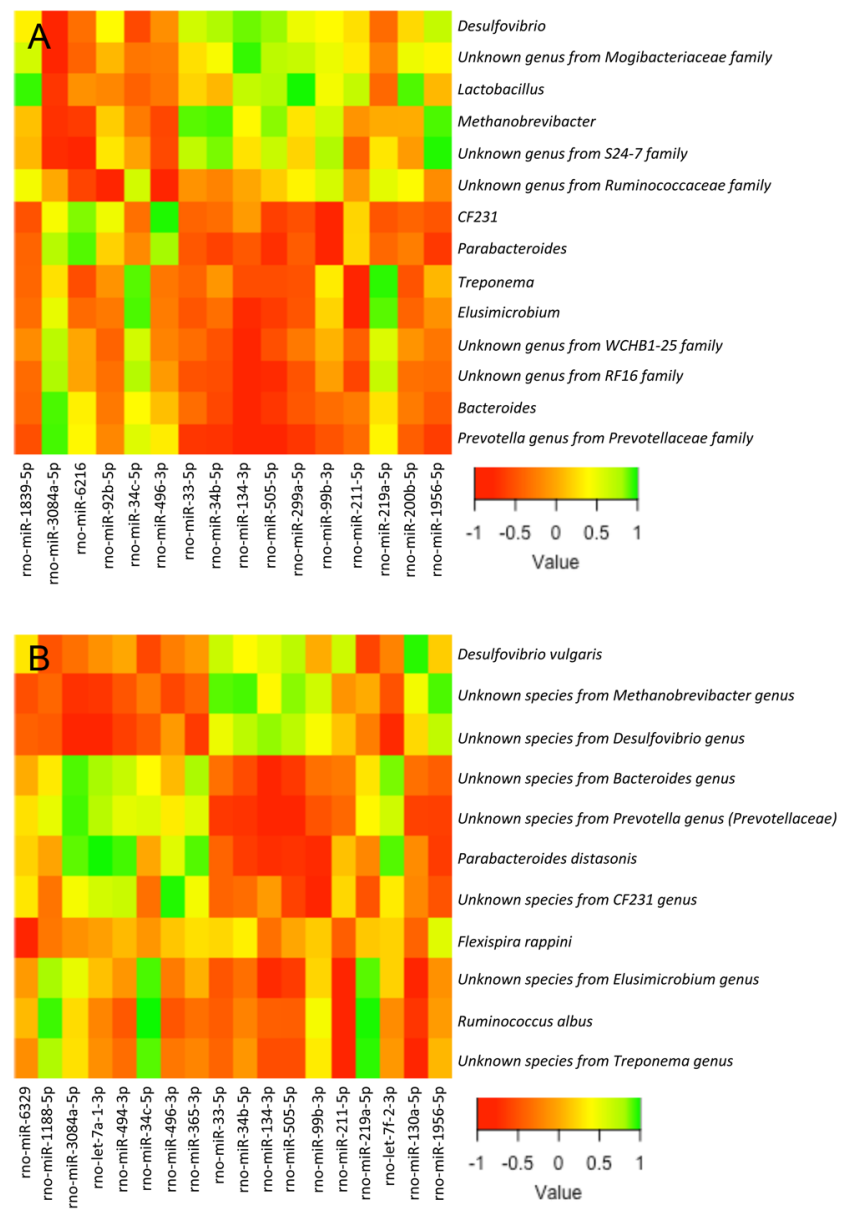

Figure 5

Heatmap from the Pearson's correlation coefficient between the bacterial genera (A) and species (B) identified by LEfSe analyses and the expression levels of the miRNAs in large intestine. A full colour version of this figure is available at https://doi.org/10.1530/JOE-20-0277.

(C) 2020 Society for Endocrinology Published by Bioscientifica Ltd. Printed in Great Britain
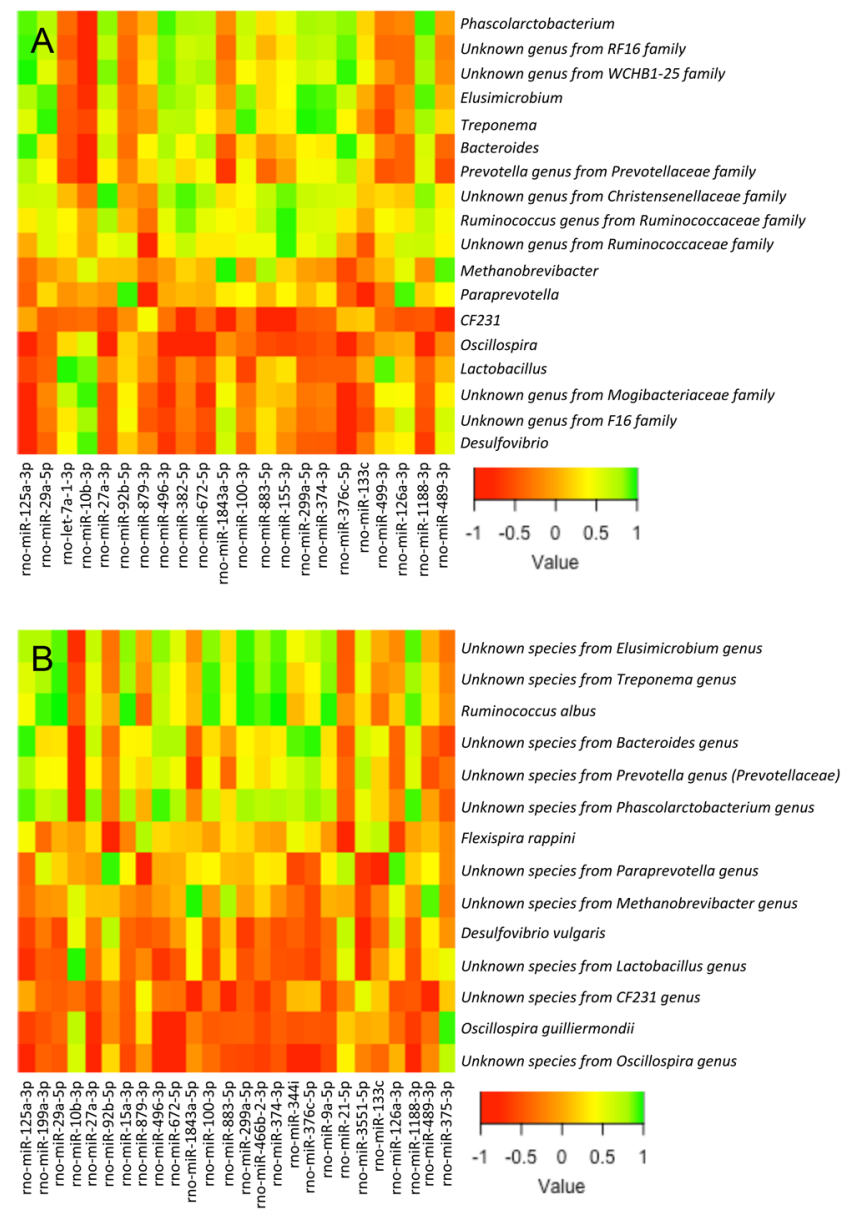

\section{Figure 6}

Heatmap from the Pearson's correlation coefficient between the bacterial genera $(A)$ and species $(B)$ identified by LEfSe analyses and the expression levels of the miRNAs in small intestine. A full colour version of this figure is available at https://doi.org/10.1530/JOE-20-0277.

Supplementary Tables 3, 4, 5, 6). Further, we performed a complementary analysis with the selected miRNAs in large and small intestine separately using the DIANAtools V.3. KEGG pathways in which selected miRNAs were assigned are shown in Tables 1 and 2, and their associated miRNAs are shown in Table 3. Of note, we found that several of the selected miRNAs were associated to (1) sex hormone-related processes: steroid biosynthesis (miR-15a-3p, miR-100-3p, miR-199a-3p), oocyte meiosis (miR-27a-3p, miR-29a-5p), progesterone-mediated oocyte maturation (miR-21-5p, miR-27a-3p, hsa-miR-34a-5p); (2) metabolism: insulin signaling pathway (miR-15a-3p, miR-27a-3p), fatty acid biosynthesis (miR-15a-3p, miR-27a-3p, miR-34a-5p, miR-34c-5p, miR-199a-3p) fatty acid metabolism (miR-15a-3p, miR-21-5p, miR-27a-3p, miR-34a-5p, miR-34c-5p, miR-92b-5p, miR-199a-3p); and (3) intestinal barrier integrity: focal adhesion (miR-27a-3p, miR-125a-3p), adherens junction 
Table 1 KEGG pathways related with miRNAs expression in the small intestine.

\section{KEGG pathway}

1. Metabolism

1.0 Global and overview maps

Fatty acid metabolism

1.1 Carbohydrate metabolism Inositol phosphate metabolism

1.3 Lipid metabolism

Fatty acid biosynthesis

$<0.001$

1.5 Amino acid metabolism Lysine degradation

1.7 Glycan biosynthesis and metabolism Mucin type O-glycan biosynthesis

Other types of O-glycan biosynthesis

0.013

Glycosaminoglycan biosynthesis - keratan sulfate

0.011

2. Genetic information processing

2.1 Transcription

Spliceosome

2.2 Translation

mRNA surveillance pathway

2.3 Folding. sorting and degradation

Protein processing in endoplasmic reticulum

$<0.001$

RNA degradation

0.041

2.4 Replication and repair

3. Environmental information processing

3.2 Signal transduction

MAPK signaling pathway

TGF-beta signaling pathway

0.009

Hippo signaling pathway

$<0.001$

TNF signaling pathway

$<0.001$

HIF-1 signaling pathway

FoxO signaling pathway

0.016

0.007

$<0.001$

Sphingolipid signaling pathway

0.002

0.047

$<0.001$

AMPK signaling pathway

mTOR signaling pathway

$<0.001$

3.3 Signaling molecules and interaction

ECM-receptor interaction

$<0.001$

4. Cellularprocesses

4.1 Transport and catabolism Endocytosis

4.2 Cell growth and death

Cell cycle

$<0.001$

0.001

Oocyte meiosis

0.003

4.3 Cellular community - eukaryotes

Focal adhesion

$<0.001$

Adherens junction

$<0.001$

Signaling pathways regulating pluripotency of stem cells

$<0.001$

4.5 Cell motility

5. Organismal systems

5.1 Immune system

Leukocyte transendothelial migration

0.034

5.2 Endocrine system

Insulin signaling pathway

$<0.001$

Thyroid hormone signaling pathway

$<0.001$

5.3 Circulatory system

Adrenergic signaling in cardiomyocytes
0.016

(Continued) https://joe.bioscientifica.com

https://doi.org/10.1530/JOE-20-0277
(C) 2020 Society for Endocrinology Published by Bioscientifica Ltd. Printed in Great Britain 
Table 1 Continued.

\begin{tabular}{|c|c|}
\hline KEGG pathway & P-value \\
\hline \multicolumn{2}{|l|}{ 5.6 Nervous system } \\
\hline Neurotrophin signaling pathway & $<0.001$ \\
\hline \multicolumn{2}{|l|}{ 5.10 Environmental adaptation } \\
\hline Circadian rhythm & 0.005 \\
\hline \multicolumn{2}{|l|}{ 6. Human diseases } \\
\hline \multicolumn{2}{|l|}{ 6.1 Cancers: overview } \\
\hline Pathways in cancer & $<0.001$ \\
\hline Transcriptional misregulation in cancer & 0.016 \\
\hline Proteoglycans in cancer & $<0.001$ \\
\hline Viral carcinogenesis & $<0.001$ \\
\hline \multicolumn{2}{|l|}{6.2 Cancers: specific types } \\
\hline Colorectal cancer & $<0.001$ \\
\hline Pancreatic cancer & 0.004 \\
\hline Glioma & 0.001 \\
\hline Thyroid cancer & $<0.001$ \\
\hline Chronic myeloid leukemia & $<0.001$ \\
\hline Renal cell carcinoma & $<0.001$ \\
\hline Prostate cancer & 0.019 \\
\hline Endometrial cancer & 0.001 \\
\hline Non-small cell lung cancer & 0.030 \\
\hline \multicolumn{2}{|l|}{ 6.4 Neurodegenerative diseases } \\
\hline Prion diseases & $<0.001$ \\
\hline \multicolumn{2}{|l|}{ 6.6 Cardiovascular diseases } \\
\hline Arrhythmogenic right ventricular cardiomyopathy (ARVC) & 0.047 \\
\hline \multicolumn{2}{|l|}{6.8 Infectious diseases: bacterial } \\
\hline Shigellosis & 0.009 \\
\hline Bacterial invasion of epithelial cells & $<0.001$ \\
\hline \multicolumn{2}{|l|}{6.9 Infectious diseases: viral } \\
\hline Hepatitis B & $<0.001$ \\
\hline Epstein-Barr virus infection & 0.004 \\
\hline \multicolumn{2}{|l|}{6.10 Infectious diseases: parasitic } \\
\hline Chagas disease (American trypanosomiasis) & 0.002 \\
\hline
\end{tabular}

(miR-27a-3p, miR-34a-5p, miR-99b-3p, miR-125a-3p, miR-130a-5p, miR-200b-5p), mucin type O-Glycan biosynthesis (miR-27a-3p, miR-29a-5p, miR-100-3p).

\section{Discussion}

Our study shows a disruption of the metabolic programming by an early (neonatal) exposure to high doses of androgen, namely testosterone propionate, in female rats. Androgenized females were heavier than intact females and displayed impaired glucose homeostasis, when concurrently exposed to an obesogenic insult (SL-HFD condition), and these phenotypic alterations were paralleled by alterations in the gut microbiota. Moreover, changes in gut microbiota were related with perturbations of miRNA expression profiles in small and large intestine, which might reflect a potential mechanism of the crosstalk between gut microbiota and host. Admittedly, our model of neonatal androgenization does not fully recapitulate the phenotype of a particular human disease.
However, it putatively shares features with human conditions linked to inappropriate exposures to androgens during early developmental periods, which include not only PCOS, for which the neonatally androgenized rat has been used previously as model (Walters et al. 2012, Romero-Ruiz et al. 2019), but also other pathologies and conditions, ranging from congenital adrenal hyperplasia to inappropriate exposures to environmental compounds with androgenic (or anti-androgenic) activity. This reinforces the translational value of our current dataset.

Sex steroids are important determinants of metabolic programming, and an inappropriate exposure to sex steroids during early maturational periods has been shown to influence metabolic homeostasis (Witchel \& Tena-Sempere 2013). In fact, it has been shown that sex steroid manipulations, and particularly postnatal androgenization, alters gut microbiota composition (Moreno-Indias et al. 2016). However, this latter study did not describe whether postnatal androgenization alters sex steroid plasma levels later on life, neither it used dynamic test to evaluate the impact of postnatal androgenization 
Table 2 KEGG pathways related with miRNAs expression in the large intestine.

\begin{tabular}{|c|c|}
\hline KEGG pathway & P-value \\
\hline \multicolumn{2}{|l|}{ 1. Metabolism } \\
\hline \multicolumn{2}{|l|}{1.0 Global and overview maps } \\
\hline 2-Oxocarboxylic acid metabolism & 0.022 \\
\hline Fatty acid metabolism & $<0.001$ \\
\hline \multicolumn{2}{|l|}{ 1.2 Energy metabolism } \\
\hline Sulfur metabolism & 0.002 \\
\hline \multicolumn{2}{|l|}{ 1.3 Lipid metabolism } \\
\hline Fatty acid biosynthesis & $<0.001$ \\
\hline \multicolumn{2}{|l|}{ 1.5 Amino acid metabolism } \\
\hline Valine. leucine and isoleucine biosynthesis & 0.001 \\
\hline \multicolumn{2}{|l|}{1.7 Glycan biosynthesis and metabolism } \\
\hline Other types of O-glycan biosynthesis & 0.002 \\
\hline Glycosphingolipid biosynthesis - lacto and neolacto series & 0.002 \\
\hline \multicolumn{2}{|l|}{ 1.8 Metabolism of cofactors and vitamins } \\
\hline Pantothenate and CoA biosynthesis & 0.027 \\
\hline \multicolumn{2}{|l|}{1.12 Chemical structure transformation maps } \\
\hline \multicolumn{2}{|l|}{ 2. Genetic information processing } \\
\hline \multicolumn{2}{|l|}{ 2.4 Replication and repair } \\
\hline \multicolumn{2}{|l|}{ 3. Environmental information processing } \\
\hline \multicolumn{2}{|l|}{ 3.2 Signal transduction } \\
\hline Hippo signaling pathway & 0.022 \\
\hline TNF signaling pathway & 0.022 \\
\hline \multicolumn{2}{|l|}{ 3.3 Signaling molecules and interaction } \\
\hline \multicolumn{2}{|l|}{ 4. Cellular processes } \\
\hline \multicolumn{2}{|l|}{ 4.3 Cellular community - eukaryotes } \\
\hline Focal adhesion & 0.042 \\
\hline Adherens junction & $<0.001$ \\
\hline \multicolumn{2}{|l|}{4.5 Cell motility } \\
\hline Regulation of actin cytoskeleton & 0.022 \\
\hline \multicolumn{2}{|l|}{ 6. Human diseases } \\
\hline \multicolumn{2}{|l|}{ 6.1 Cancers:overview } \\
\hline Pathways in cancer & 0.022 \\
\hline \multicolumn{2}{|l|}{6.2 Cancers: specific types } \\
\hline Glioma & 0.020 \\
\hline \multicolumn{2}{|l|}{6.8 Infectious diseases: bacterial } \\
\hline Pathogenic Escherichia coli infection & 0.007 \\
\hline Salmonella infection & 0.016 \\
\hline Shigellosis & 0.005 \\
\hline Bacterial invasion of epithelial cells & 0.003 \\
\hline
\end{tabular}

on glucose homeostasis. More importantly, this previous study did not include analyses in males or at two different age-points; the latter have allowed us to highlight important temporal changes in microbiota composition between early and late-adulthood. Finally, the previous study did not analyze the effect of postnatal androgenization on gut microbiota under lean (NL-CD) and obese (SL-HFD) condition separately, as in that former study the groups of androgenized females under NL-CD and SL-HFD conditions were merged for microbiota analyses.

Notably, our study showed that whereas androgenization had no effect on endogenous androgens levels later in life (except for a slight reduction on the androstenedione levels), it caused an overt alteration of serum E2 and E1 levels, in addition to reduced levels of progesterone. Albeit the positive role of estrogen receptor activation on insulin sensitivity has been described in different tissues (Yan et al. 2019), it has been pointed out also that the constant maintenance of high estrogen levels could lead to insulin resistance, as a consequence of increasing insulin secretion (Polderman et al. 1994, Alonso-Magdalena et al. 2006). This suggests that the increased serum E2 and E1 levels might contribute to the lower insulin sensitivity observed in androgenized females compared to the intact adult females, an effect exacerbated under SL-HFD condition.

Despite the proven anti-obesity effects of estrogens, through decreasing food intake and increasing energy expenditure in females (Eckel 2011, Xu et al. 2019), 
Table 3 KEGG pathways associated with microbiome-related miRNAs.

\section{KEGG pathway}

2-Oxocarboxylic acid metabolism (hsa01210)

Adherens junction (hsa04520)

Allograft rejection (hsa05330)

alpha-Linolenic acid metabolism (hsa00592)

Amoebiasis (hsa05146)

AMPK signaling pathway (hsa04152)

Antigen processing and presentation (hsa04612)

Apoptosis (hsa04210)

Arrhythmogenic right ventricular cardiomyopathy

(ARVC) (hsa05412)

Axon guidance (hsa04360)

Bacterial invasion of epithelial cells (hsa05100)

Biosynthesis of unsaturated fatty acids (hsa01040)

Bladder cancer (hsa05219)

Cell cycle (hsa04110)

Central carbon metabolism in cancer (hsa05230)

Chronic myeloid leukemia (hsa05220)

Circadian entrainment (hsa04713)

Colorectal cancer (hsa05210)

Cytokine-cytokine receptor interaction (hsa04060)

D-Glutamine and D-glutamate metabolism (hsa00471)

ECM-receptor interaction (hsa04512)

Endometrial cancer (hsa05213)

ErbB signaling pathway (hsa04012)

Ether lipid metabolism (hsa00565)

Fatty acid biosynthesis (hsa00061)

Fatty acid degradation (hsa00071)

Fatty acid elongation (hsa00062)

Fatty acid metabolism (hsa01212)

Focal adhesion (hsa04510)

FoxO signaling pathway (hsa04068)

Glioma (hsa05214)

Glycosphingolipid biosynthesis - lacto and neolacto series (hsa00601)

Graft-vs-host disease (hsa05332)

Hepatitis B (hsa05161)

Hippo signaling pathway (hsa04390)

HTLV-I infection (hsa05166)

Insulin signaling pathway (hsa04910)

Leukocyte transendothelial migration (hsa04670)

Lysine degradation (hsa00310)

Melanoma (hsa05218)

mRNA surveillance pathway (hsa03015)

mTOR signaling pathway (hsa04150)

Mucin type O-Glycan biosynthesis (hsa00512)

Neurotrophin signaling pathway (hsa04722)

NF-kappa B signaling pathway (hsa04064)

Non-homologous end-joining (hsa03450)

Non-small cell lung cancer (hsa05223)

One carbon pool by folate (hsa00670)

Oocyte meiosis (hsa04114)

Other types of O-glycan biosynthesis (hsa00514)

p53 signaling pathway (hsa04115)

Pancreatic cancer (hsa05212)

\section{Human miRNA}

hsa-miR-505-5p-L

hsa-miR-99b-3p-SL; hsa-miR-200b-5p-L; hsa-miR-130a-5p-L;

hsa-miR-125a-3p-S; hsa-miR-27a-3p-S

hsa-miR-10b-3p-S; hsa-miR-92b-5p-SL; hsa-miR-382-5p-S

hsa-miR-92b-5p-SL

hsa-miR-382-5p-S

hsa-miR-27a-3p-S; hsa-miR-15a-3p-S

hsa-miR-10b-3p-S; hsa-miR-92b-5p-SL; hsa-miR-382-5p-S; hsa-miR-211-5p-L

hsa-miR-200b-5p-L

hsa-miR-100-3p-S; hsa-miR-505-5p-L

hsa-miR-505-5p-L; hsa-miR-27a-3p-S

hsa-miR-99b-3p-SL; hsa-miR-27a-3p-S; hsa-miR-21-5p-S

hsa-miR-21-5p-S

hsa-miR-27a-3p-S

hsa-miR-99b-3p-SL; hsa-miR-27a-3p-S; hsa-miR-21-5p-S

hsa-miR-34c-5p-L; hsa-miR-382-5p-S; hsa-miR-125a-3p-S; hsa-miR-27a-3p-S

hsa-miR-27a-3p-S; hsa-miR-15a-3p-S

hsa-miR-10b-3p-S; hsa-miR-200b-5p-L

hsa-miR-125a-3p-S; hsa-miR-27a-3p-S; hsa-miR-15a-3p-S; hsa-miR-21-5p-S

hsa-miR-211-5p-L

hsa-miR-200b-5p-L

hsa-miR-382-5p-S; hsa-miR-100-3p-S; hsa-miR-211-5p-L;

hsa-miR-130a-5p-L; hsa-miR-125a-3p-S; hsa-miR-27a-3p-S

hsa-miR-27a-3p-S; hsa-miR-21-5p-S

hsa-miR-27a-3p-s

hsa-miR-100-3p-S

hsa-miR-34c-5p-L; hsa-miR-199a-3p-S; hsa-miR-27a-3p-S; hsa-miR-15a-3p-S

hsa-miR-92b-5p-SL; hsa-miR-29a-5p-S; hsa-miR-21-5p-S

hsa-miR-29a-5p-S; hsa-miR-21-5p-S

hsa-miR-92b-5p-SL; hsa-miR-34c-5p-L; hsa-miR-199a-3p-S;

hsa-miR-27a-3p-S; hsa-miR-15a-3p-S; hsa-miR-21-5p-S

hsa-miR-125a-3p-S; hsa-miR-27a-3p-S

hsa-miR-125a-3p-S; hsa-miR-27a-3p-S; hsa-miR-21-5p-S

hsa-miR-34c-5p-L; hsa-miR-27a-3p-S; hsa-miR-15a-3p-S

hsa-miR-505-5p-L; hsa-miR-130a-5p-L

hsa-miR-10b-3p-S

hsa-miR-27a-3p-S; hsa-miR-15a-3p-S; hsa-miR-21-5p-S

hsa-miR-99b-3p-SL; hsa-miR-29a-5p-S; hsa-miR-27a-3p-S; hsa-miR-21-5p-S

hsa-miR-15a-3p-S

hsa-miR-27a-3p-S; hsa-miR-15a-3p-S

hsa-miR-200b-5p-L

hsa-miR-505-5p-L; hsa-miR-125a-3p-S; hsa-miR-27a-3p-S; hsa-miR-21-5p-S

hsa-miR-27a-3p-S

hsa-miR-29a-5p-S

hsa-miR-382-5p-S; hsa-miR-27a-3p-S

hsa-miR-100-3p-S; hsa-miR-29a-5p-S; hsa-miR-27a-3p-S

hsa-miR-27a-3p-S

hsa-miR-200b-5p-L

hsa-miR-99b-3p-SL

hsa-miR-27a-3p-S

hsa-miR-125a-3p-S

hsa-miR-29a-5p-S; hsa-miR-27a-3p-S

hsa-miR-505-5p-L

hsa-miR-27a-3p-S; hsa-miR-21-5p-S

hsa-miR-27a-3p-S 
Table 3 Continued.

\begin{tabular}{l}
\hline KEGG pathway \\
\hline Pantothenate and CoA biosynthesis (hsa00770) \\
Parkinson's disease (hsa05012) \\
Pathways in cancer (hsa05200) \\
PI3K-Akt signaling pathway (hsa04151) \\
Prion diseases (hsa05020) \\
Progesterone-mediated oocyte maturation (hsa04914) \\
Prolactin signaling pathway (hsa04917) \\
Prostate cancer (hsa05215) \\
Protein processing in endoplasmic reticulum \\
(hsa04141) \\
Proteoglycans in cancer (hsa05205) \\
Renal cell carcinoma (hsa05211) \\
Salmonella infection (hsa05132) \\
Shigellosis (hsa05131) \\
Signaling pathways regulating pluripotency of stem \\
cells (hsa04550) \\
Sphingolipid metabolism (hsa00600) \\
Sphingolipid signaling pathway (hsa04071) \\
Steroid biosynthesis (hsa00100) \\
Sulfur metabolism (hsa00920) \\
Taste transduction (hsa04742) \\
TGF-beta signaling pathway (hsa04350) \\
Thyroid cancer (hsa05216) \\
Thyroid hormone signaling pathway (hsa04919) \\
Transcriptional misregulation in cancer (hsa05202) \\
Ubiquitin mediated proteolysis (hsa04120) \\
Valine, leucine and isoleucine biosynthesis (hsa00290) \\
Viral carcinogenesis (hsa05203) \\
Viral myocarditis (hsa05416) \\
Pal
\end{tabular}

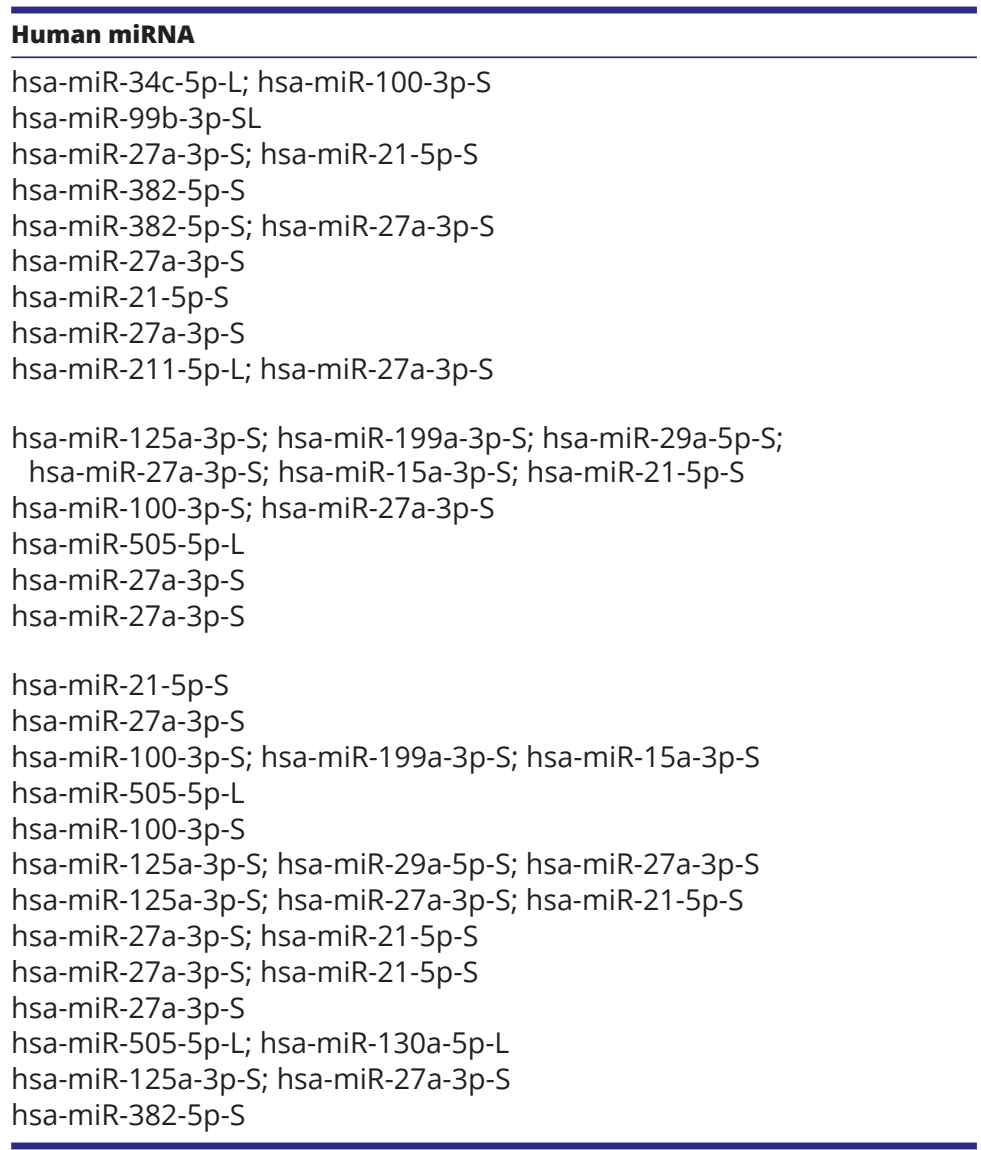

hsa, Homo sapiens; L, large intestine; S, small intestine.

our study shows that androgenized females, which presented higher estrogen levels, were heavier than intact females, suggesting the contribution of another factors or mediators for such an obese phenotype. These might include changes in gut microbiota, as it has been previously shown that the structure of gut microbiota is altered in parallel to changes on sex hormones levels (Markle et al. 2013, Insenser et al. 2018, Santos-Marcos et al. 2018). However, the causality between these two phenomena has not been described so far. Our study conclusively documents that early androgenization of females alters the gut microbiota profile, evidenced by the fact that the gut microbiota under NL-CD condition from androgenized females at PND-50 was characterized by higher Bacteroidetes and lower Firmicutes than the intact females. However, differences in these major phyla disappeared in older rats (PND-150), while in those androgenization reduced the abundance of Actinobacteria and Euryarchaeota and increased Cyanobacteria.

Different hormonal changes take place through the lifespan (Horstman et al. 2012). For example, major hormonal changes occur during puberty (Koolschijn et al. 2014), which may also influence gut microbiota composition (Kim et al. 2019). Our study showed that the differences between androgenized females and intact females were more exacerbated at early adulthood (PND-50) than later in life (late adulthood at PND-150). In fact, the differences observed between androgenized and intact females under NL-CD at PND-50 in the major phyla, Bacteroidetes and Firmicutes, were not observed at PND-150. These differences also disappeared at early adulthood (PND-50) when animals were overfed, suggesting an additional effect of obesity, reducing the differences in gut microbiota between androgenized and intact females, as observed later in life. However, postnatal overfeeding increased the $\mathrm{F} / \mathrm{B}$ ratio in both androgenized and intact females as expected taking into account that the $\mathrm{F} / \mathrm{B}$ ratio, which has great importance in the development of obesity, is commonly increased in this condition (Turnbaugh et al. 2006). Moreover, postnatal overfeeding reduced the alterations caused by androgenization in gut microbiota in the late adulthood 
(PND-150), with only four genera differing between groups, but androgenized females were characterized by higher Bacteroidetes than intact females. Overall, our study supports the idea that the interaction of the hormonal status during the lifespan with the nutritional conditions might modulate gut microbiota.

As intestinal miRNAs are increasingly recognized as potential decoders of dysbiosis into cardiometabolic diseases, we also explored the putative cross-talk between gut microbiota and host through regulation of miRNA expression in small and large intestine (Liu et al. 2016, Serino 2016). Of note, we did not include in the analysis all the bacterial taxa but only those identified by LEfSe analysis in order to reduce the random association. This approach allowed us to detect those miRNAs that were affected by neonatal androgenization, and changed in parallel with changes in bacterial taxa, identified by LEfSe analysis. Among those, we could identify the miRNAs that potentially mediate the interaction between sex hormones, gut microbiota and host metabolism.

In addition to several KEGG pathways related with metabolism, such as fatty acid metabolism or sphingolipid signaling pathway, which in turn are related with the development of type 2 diabetes mellitus (Imamura et al. 2017) and cardiovascular disease (Razquin et al. 2018), our approach detected also potential miRNA-mediated relationships between the gut microbiota, metabolism and sex steroid-related pathways. For example, the expression levels of miR-27a-3p were positively related with Oscillospira, a short chain fatty acids (SCFA)producer bacterial genus (Gophna et al. 2017), which in turn may be related with glucose homeostasis, as SCFA increases the action and release of insulin through intestinal incretins (Freeland \& Wolever 2010, Tarini \& Wolever 2010). Moreover, SCFAs are also involved in energy metabolism and appetite regulation through the hormones leptin and ghrelin (Shen et al. 2013, Byrne et al. 2015, Lv et al. 2018), whose plasma levels were altered in our study and may be at least partially responsible of the weight gain in androgenized females. In addition, miR-27a-3p is involved in fatty acid metabolism, insulin signaling, oocyte maturation, in addition to contribute to the intestinal barrier integrity by processes such as adherens junction, focal adhesion and mucin type O-glycan biosynthesis. In addition, other miRNAs showed by this study, such as miR-29a-5p, and miR-100-3p, may also link metabolism and sex hormone actions on the basis of their previously reported involvement in mediating sex-steroid actions in other tissues, such as progesterone-mediated oocyte maturation
(Navakanitworakul et al. 2016, Tesfaye et al. 2018, Tu et al. 2019). These evidences suggest that the cross-talk between gut microbiota and the host via specific miRNAs may involve also gonadal steroid mediated events. In addition, the expression of these miRNAs was related with the intestinal abundance of Treponema, a bacterial genus that enhances the ability to extract calories from resistant starch and oligosaccharides, as well as carbohydrates that escape digestion in the small intestine and are fermented in the gut (Flint et al. 2008).

Taken together, our results support the idea that changes in the gut microbiome composition, in response to alterations in sex hormones, may trigger a further gene expression response in the host for selective gut microbial colonization, via miRNAs as a mediator of this process. Thus, the potential contribution of intestinal bacteria to the expression levels of miRNAs as a gut microbiotaintestine cross-talk system, in line with previous studies (Liu et al. 2016, Serino 2016), would link changes in the gut microbiota composition to processes such as metabolism and sex hormone metabolic programming.

Our study suggests that nutritional and hormonal disruption in early periods of the development alters metabolic programming with durable consequences later in life. Moreover, this disruption dynamically affects the structure of gut microbiota, currently considered as an integrated organ, which may interact with the host via a cross-talk mediated by intestinal miRNAs, which may serve as an additional link between early mal-programming and impaired metabolic health later in life.

\section{Supplementary materials}

This is linked to the online version of the paper at https://doi.org/10.1530/ JOE-20-0277.

\section{Declaration of interest}

The authors declare that there is no conflict of interest that could be perceived as prejudicing the impartiality of the research reported.

\section{Funding}

This work was supported by research grants from Ministerio de Economia y Competitividad (CP14/00114 to A C; PIE14/00005 to J L-M and M T-S; AGL2015-67896-P to J L-M and A C); Ministerio de Ciencia e Innovacion (FIS PI19/00299 to A C; DTS19/00007 to A C; PI16/01777 to P P M); Fondo Europeo de Desarrollo Regional (FEDER). A C is supported by an ISCIII research contract, Instituto de Salud Carlos III (Programa Miguel Servet CP14/00114 and CPII19/00007).

\section{Authors contribution statement}

A Barroso and Jose A Santos-Marcos contributed to the animal experiments, acquisition, analysis and interpretation of data, and drafting of the 
manuscript. C Perdices-Lopez, A Vega-Rojas, M A Sanchez-Garrido, and Y Krylova contributed to the animal experiments, analysis and interpretation of data. $\mathrm{H}$ Molina-Abril contributed to analysis and interpretation of data. C Ohlsson, P Perez Martinez, M Poutanen, J Lopez-Miranda contributed to interpretation of data and edited the manuscript. M Tena-Sempere and A Camargo designed and conducted the experiments, approval of the manuscript and are the guarantor of this work and, as such, had full access to all the data in the study and takes responsibility for the integrity of the data and the accuracy of the data analysis.

\section{Acknowledgements}

The CIBEROBN is an initiative of the Instituto de Salud Carlos III, Madrid, Spain.

\section{References}

Alonso-Magdalena P, Morimoto S, Ripoll C, Fuentes E \& Nadal A 2006 The estrogenic effect of bisphenol A disrupts pancreatic beta-cell function in vivo and induces insulin resistance. Environmental Health Perspectives 114 106-112. (https://doi.org/10.1289/ehp.8451)

Bokulich NA, Kaehler BD, Rideout JR, Dillon M, Bolyen E, Knight R, Huttley GA \& Gregory Caporaso J 2018 Optimizing taxonomic classification of marker-gene amplicon sequences with QIIME 2's q2-feature-classifier plugin. Microbiome 6 90. (https://doi.org/10.1186/ s40168-018-0470-z)

Bolyen E, Rideout JR, Dillon MR, Bokulich NA, Abnet CC, Al-Ghalith GA, Alexander H, Alm EJ, Arumugam M, Asnicar F, et al. 2019 Reproducible, interactive, scalable and extensible microbiome data science using QIIME 2. Nature Biotechnology 37 852-857. (https://doi. org/10.1038/s41587-019-0209-9)

Byrne CS, Chambers ES, Morrison DJ \& Frost G 2015 The role of short chain fatty acids in appetite regulation and energy homeostasis. International Journal of Obesity 39 1331-1338. (https://doi. org/10.1038/ijo.2015.84)

Callahan BJ, McMurdie PJ, Rosen MJ, Han AW, Johnson AJ \& Holmes SP 2016 DADA2: high-resolution sample inference from Illumina amplicon data. Nature Methods 13 581-583. (https://doi.org/10.1038/ nmeth.3869)

Castellano JM, Bentsen AH, Sanchez-Garrido MA, Ruiz-Pino F, Romero M, Garcia-Galiano D, Aguilar E, Pinilla L, Dieguez C, Mikkelsen JD, et al. 2011 Early metabolic programming of puberty onset: impact of changes in postnatal feeding and rearing conditions on the timing of puberty and development of the hypothalamic kisspeptin system. Endocrinology 152 3396-3408. (https://doi.org/10.1210/en.2010-1415)

Cross TL, Kasahara K \& Rey FE 2018 Sexual dimorphism of cardiometabolic dysfunction: gut microbiome in the play? Molecular Metabolism 15 70-81. (https://doi.org/10.1016/j.molmet.2018.05.016)

Eckel LA 2011 The ovarian hormone estradiol plays a crucial role in the control of food intake in females. Physiology and Behavior $\mathbf{1 0 4}$ 517-524. (https://doi.org/10.1016/j.physbeh.2011.04.014)

Faulkner JL \& Belin de Chantemele EJ 2019 Sex hormones, aging and cardiometabolic syndrome. Biology of Sex Differences 10 30. (https:// doi.org/10.1186/s13293-019-0246-6)

Flint HJ, Bayer EA, Rincon MT, Lamed R \& White BA 2008 Polysaccharide utilization by gut bacteria: potential for new insights from genomic analysis. Nature Reviews: Microbiology 6 121-131. (https://doi. org/10.1038/nrmicro1817)

Freeland KR \& Wolever TM 2010 Acute effects of intravenous and rectal acetate on glucagon-like peptide-1, peptide YY, ghrelin, adiponectin and tumour necrosis factor-alpha. British Journal of Nutrition 103 460-466. (https://doi.org/10.1017/S0007114509991863)

Gophna U, Konikoff T \& Nielsen HB 2017 Oscillospira and related bacteria - from metagenomic species to metabolic features. Environmental Microbiology 19 835-841. (https://doi. org/10.1111/1462-2920.13658)
Hammer Ø, Harper DAT \& Ryan PD 2001 PAST: paleontological statistics software package for education and data analysis. Palaeontologia Electronica 49.

Horstman AM, Dillon EL, Urban RJ \& Sheffield-Moore M 2012 The role of androgens and estrogens on healthy aging and longevity. Journals of Gerontology: Series A, Biological Sciences and Medical Sciences 67 1140-1152. (https://doi.org/10.1093/gerona/gls068)

Imamura F, Sharp SJ, Koulman A, Schulze MB, Kroger J, Griffin JL, Huerta JM, Guevara M, Sluijs I, Agudo A, et al. 2017 A combination of plasma phospholipid fatty acids and its association with incidence of type 2 diabetes: the EPIC-InterAct case-cohort study. PLoS Medicine 14 e1002409. (https://doi.org/10.1371/journal. pmed.1002409)

Insenser M, Murri M, Del Campo R, Martinez-Garcia MÁ, FernandezDuran E \& Escobar-Morreale HF 2018 Gut microbiota and the polycystic ovary syndrome: influence of sex, sex hormones, and obesity. Journal of Clinical Endocrinology and Metabolism 103 2552-2562. (https://doi.org/10.1210/jc.2017-02799)

Jie Z, Xia H, Zhong SL, Feng Q, Li S, Liang S, Zhong H, Liu Z, Gao Y, Zhao H, et al. 2017 The gut microbiome in atherosclerotic cardiovascular disease. Nature Communications 8 845. (https://doi. org/10.1038/s41467-017-00900-1)

Kim YS, Unno T, Kim BY \& Park MS 2019 Sex differences in gut microbiota. World Journal of Men's Health 38 48-60. (https://doi. org/10.5534/wjmh.190009)

Klindworth A, Pruesse E, Schweer T, Peplies J, Quast C, Horn M \& Glockner FO 2013 Evaluation of general 16S ribosomal RNA gene PCR primers for classical and next-generation sequencing-based diversity studies. Nucleic Acids Research 41 e1. (https://doi.org/10.1093/nar/ gks808)

Koolschijn PC, Peper JS \& Crone EA 2014 The influence of sex steroids on structural brain maturation in adolescence. PLOS ONE 9 e83929. (https://doi.org/10.1371/journal.pone.0083929)

Liu S, da Cunha AP, Rezende RM, Cialic R, Wei Z, Bry L, Comstock LE, Gandhi R \& Weiner HL 2016 The host shapes the gut microbiota via fecal microRNA. Cell Host and Microbe 19 32-43. (https://doi. org/10.1016/j.chom.2015.12.005)

Lozupone C \& Knight R 2005 UniFrac: a new phylogenetic method for comparing microbial communities. Applied and Environmental Microbiology 71 8228-8235. (https://doi.org/10.1128/AEM.71.12.82288235.2005)

Lv Y, Liang T, Wang G \& Li Z 2018 Ghrelin, a gastrointestinal hormone, regulates energy balance and lipid metabolism. Bioscience Reports 38 BSR20181061. (https://doi.org/10.1042/BSR20181061)

Markle JG, Frank DN, Mortin-Toth S, Robertson CE, Feazel LM, RolleKampczyk U, von Bergen M, McCoy KD, Macpherson AJ \& Danska JS 2013 Sex differences in the gut microbiome drive hormonedependent regulation of autoimmunity. Science 339 1084-1088. (https://doi.org/10.1126/science.1233521)

Mauvais-Jarvis F 2015 Sex differences in metabolic homeostasis, diabetes, and obesity. Biology of Sex Differences 6 14. (https://doi.org/10.1186/ s13293-015-0033-y)

Mauvais-Jarvis F, Clegg DJ \& Hevener AL 2013 The role of estrogens in control of energy balance and glucose homeostasis. Endocrine Reviews 34 309-338. (https://doi.org/10.1210/er.2012-1055)

McDonald D, Price MN, Goodrich J, Nawrocki EP, DeSantis TZ, Probst A, Andersen GL, Knight R \& Hugenholtz P 2012 An improved Greengenes taxonomy with explicit ranks for ecological and evolutionary analyses of bacteria and archaea. ISME Journal 6 610-618. (https://doi.org/10.1038/ismej.2011.139)

Moreno-Indias I, Sanchez-Alcoholado L, Sanchez-Garrido MÁ, MartinNunez GM, Perez-Jimenez F, Tena-Sempere M, Tinahones FJ \& Queipo-Ortuno MI 2016 Neonatal androgen exposure causes persistent gut microbiota dysbiosis related to metabolic disease in adult female rats. Endocrinology 157 4888-4898. (https://doi org/10.1210/en.2016-1317) https://joe.bioscientifica.com

https://doi.org/10.1530/JOE-20-0277 (c) 2020 Society for Endocrinology Published by Bioscientifica Ltd. Printed in Great Britain 
Navakanitworakul R, Hung WT, Gunewardena S, Davis JS, Chotigeat W \& Christenson LK 2016 Characterization and small RNA content of extracellular vesicles in follicular fluid of developing bovine antral follicles. Scientific Reports 6 25486. (https://doi.org/10.1038/ srep25486)

Nilsson ME, Vandenput L, Tivesten Å, Norlen AK, Lagerquist MK, Windahl SH, Borjesson AE, Farman HH, Poutanen M, Benrick A, et al. 2015 Measurement of a comprehensive sex steroid profile in rodent serum by high-sensitive gas chromatography-tandem mass spectrometry. Endocrinology 156 2492-2502. (https://doi.org/10.1210/ en.2014-1890)

Pinilla L, Barreiro ML, Gonzalez LC, Tena-Sempere M \& Aguilar E 2002 Comparative effects of testosterone propionate, oestradiol benzoate, ICI 182,780, tamoxifen and raloxifene on hypothalamic differentiation in the female rat. Journal of Endocrinology 172 441-448. (https://doi.org/10.1677/joe.0.1720441)

Polderman KH, Gooren LJ, Asscheman H, Bakker A \& Heine RJ 1994 Induction of insulin resistance by androgens and estrogens. Journal of Clinical Endocrinology and Metabolism 79 265-271. (https://doi. org/10.1210/jcem.79.1.8027240)

Rao PM, Kelly DM \& Jones TH 2013 Testosterone and insulin resistance in the metabolic syndrome and T2DM in men. Nature Reviews: Endocrinology 9 479-493. (https://doi.org/10.1038/ nrendo.2013.122)

Razquin C, Liang L, Toledo E, Clish CB, Ruiz-Canela M, Zheng Y, Wang DD, Corella D, Castaner O, Ros E, et al. 2018 Plasma lipidome patterns associated with cardiovascular risk in the PREDIMED trial: a case-cohort study. International Journal of Cardiology 253 126-132. (https://doi.org/10.1016/j.ijcard.2017.10.026)

Ritchie ME, Silver J, Oshlack A, Holmes M, Diyagama D, Holloway A \& Smyth GK 2007 A comparison of background correction methods for two-colour microarrays. Bioinformatics 23 2700-2707. (https://doi. org/10.1093/bioinformatics/btm412)

Romero-Ruiz A, Skorupskaite K, Gaytan F, Torres E, Perdices-Lopez C, Mannaerts BM, Qi S, Leon S, Manfredi-Lozano M, Lopez-Rodriguez C, et al. 2019 Kisspeptin treatment induces gonadotropic responses and rescues ovulation in a subset of preclinical models and women with polycystic ovary syndrome. Human Reproduction 34 2495-2512. (https://doi.org/10.1093/humrep/dez205)

Sanchez-Garrido MA, Castellano JM, Ruiz-Pino F, Garcia-Galiano D, Manfredi-Lozano M, Leon S, Romero-Ruiz A, Dieguez C, Pinilla L \& Tena-Sempere M 2013 Metabolic programming of puberty: sexually dimorphic responses to early nutritional challenges. Endocrinology 154 3387-3400. (https://doi.org/10.1210/en.2012-2157)

Sanchez-Garrido MA, Ruiz-Pino F, Manfredi-Lozano M, Leon S, GarciaGaliano D, Castano JP, Luque RM, Romero-Ruiz A, Castellano JM, Dieguez C, et al. 2014 Obesity-induced hypogonadism in the male: premature reproductive neuroendocrine senescence and contribution of Kiss1-mediated mechanisms. Endocrinology 155 1067-1079. (https://doi.org/10.1210/en.2013-1584)

Santos-Marcos JA, Rangel-Zuniga OA, Jimenez-Lucena R, QuintanaNavarro GM, Garcia-Carpintero S, Malagon MM, Landa BB, TenaSempere M, Perez-Martinez P, Lopez-Miranda J, et al. 2018 Influence of gender and menopausal status on gut microbiota. Maturitas 116 43-53. (https://doi.org/10.1016/j.maturitas.2018.07.008)

Segata N, Izard J, Waldron L, Gevers D, Miropolsky L, Garrett WS \& Huttenhower C 2011 Metagenomic biomarker discovery and explanation. Genome Biology 12 R60. (https://doi.org/10.1186/ gb-2011-12-6-r60)

Serino M 2016 MicroRNAs: decoders of dysbiosis into metabolic diseases? Journal of Diabetes and Metabolism 7 698. (https://doi. org/10.4172/2155-6156.1000698)
Shen J, Obin MS \& Zhao L 2013 The gut microbiota, obesity and insulin resistance. Molecular Aspects of Medicine 34 39-58. (https://doi. org/10.1016/j.mam.2012.11.001)

Smyth GK 2005 Limma: linear models for microarray data. In Bioinformatics and Computational Biology Solutions Using $R$ and Bioconductor. Statistics for Biology and Health. Eds R Gentleman, W, Huber, RA, Irizarry \& S, Dudoit. New York, NY, USA: Springer.

Tarini J \& Wolever TM 2010 The fermentable fibre inulin increases postprandial serum short-chain fatty acids and reduces free-fatty acids and ghrelin in healthy subjects. Applied Physiology, Nutrition, and Metabolism 35 9-16. (https://doi.org/10.1139/H09-119)

Tesfaye D, Gebremedhn S, Salilew-Wondim D, Hailay T, Hoelker M, Grosse-Brinkhaus C \& Schellander K 2018 MicroRNAs: tiny molecules with a significant role in mammalian follicular and oocyte development. Reproduction 155 R121-R135. (https://doi.org/10.1530/ REP-17-0428)

Tremaroli V \& Backhed F 2012 Functional interactions between the gut microbiota and host metabolism. Nature 489 242-249. (https://doi. org/10.1038/nature11552)

Tu J, Cheung AH, Chan CL \& Chan WY 2019 The role of microRNAs in ovarian granulosa cells in health and disease. Frontiers in Endocrinology 10 174. (https://doi.org/10.3389/fendo.2019.00174)

Turnbaugh PJ, Ley RE, Mahowald MA, Magrini V, Mardis ER \& Gordon JI 2006 An obesity-associated gut microbiome with increased capacity for energy harvest. Nature $\mathbf{4 4 4}$ 1027-1031. (https://doi.org/10.1038/ nature05414)

Velasco I, Leon S, Barroso A, Ruiz-Pino F, Heras V, Torres E, Leon M, Ruohonen ST, Garcia-Galiano D, Romero-Ruiz A, et al. 2019 Gonadal hormone-dependent vs. -independent effects of kisspeptin signaling in the control of body weight and metabolic homeostasis. Metabolism: Clinical and Experimental 98 84-94. (https://doi.org/10.1016/j. metabol.2019.06.007)

Vlachos IS, Zagganas K, Paraskevopoulou MD, Georgakilas G, Karagkouni D, Vergoulis T, Dalamagas T \& Hatzigeorgiou AG 2015 Diana-miRPath v3.0: deciphering microRNA function with experimental support. Nucleic Acids Research 43 W460-W466. (https:// doi.org/10.1093/nar/gkv403)

Walters KA, Allan CM \& Handelsman DJ 2012 Rodent models for human polycystic ovary syndrome. Biology of Reproduction 86 149, 1-149, 12. (https://doi.org/10.1095/biolreprod.111.097808)

Witchel SF \& Tena-Sempere M 2013 The Kiss1 system and polycystic ovary syndrome: lessons from physiology and putative pathophysiologic implications. Fertility and Sterility 100 12-22. (https://doi.org/10.1016/j.fertnstert.2013.05.024)

Witchel SF, Oberfield SE \& Peña AS 2019, Polycystic ovary syndrome: pathophysiology, presentation, and treatment with emphasis on adolescent girls. Journal of the Endocrine Society 3 1545-1573. (https:// doi.org/10.1210/js.2019-00078)

Xu Y, Nedungadi TP, Zhu L, Sobhani N, Irani BG, Davis KE, Zhang X, Zou F, Gent LM, Hahner LD, et al. 2019 Distinct hypothalamic neurons mediate estrogenic effects on energy homeostasis and reproduction. Cell Metabolism 29 1232. (https://doi.org/10.1016/j. cmet.2019.04.006)

Yan H, Yang W, Zhou F, Li X, Pan Q, Shen Z, Han G, Newell-Fugate A, Tian Y, Majeti R, et al. 2019 Estrogen improves insulin sensitivity and suppresses gluconeogenesis via the transcription factor FoxO1 Diabetes 68 291-304. (https://doi.org/10.2337/db18-0638)

Yang YH, Dudoit S, Luu P \& Speed TP 2001 Normalization for cDNA microarraydata. In SPIE Proceedings Vol. 4266: Microarrays: Optical Technologies and Informatics, pp. 141-152. Eds YCML Bittner, AN Dorsel \& ER Dougherty. San Jose, CA, USA: SPIE. (https://doi. org/10.1117/12.427982)

Received in final form 28 July 2020

Accepted 4 August 2020

Accepted Manuscript published online 4 August 2020 https://joe.bioscientifica.com https://doi.org/10.1530/JOE-20-0277 (c) 2020 Society for Endocrinology Published by Bioscientifica Ltd. Printed in Great Britain 